\title{
Managing the second stage of labor: An evidence-based approach
}

\author{
Continuously assessing and monitoring maternal, fetal, and modifiable \\ factors in the second stage of labor may aid clinicians in weighing the \\ appropriateness of expectant management against operative delivery
}

\author{
Jeny Ghartey, DO, MS, and Alison G. Cahill, MD, MSCI
}

\section{IN THIS ARTICLE}

Ideal labor duration page 41

Assessing labor progress

page 42

Immediate vs delayed pushing page 43

\begin{abstract}
CASE Woman in second stage with prolonged pushing

Ms. J. is an 18-year-old woman (G1P0) at 39 weeks' gestation whose cervix is completely dilated; she has been actively pushing for 60 minutes. The estimated fetal weight is $8 \mathrm{lb}$, and electronic fetal monitoring shows a Category I fetal heart rate (FHR) tracing. The presenting part remains at 0 station and occiput transverse despite great pushing effort.
\end{abstract}

After another hour of active pushing, the FHR becomes Category II with repetitive variable decelerations. During the third hour of the second stage, Ms. J. is diagnosed with chorioamnionitis and the fetus remains at 0 station. She undergoes a primary cesarean delivery (CD) complicated by bilateral lower uterine

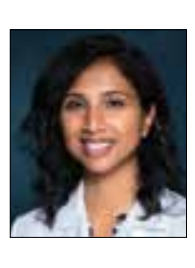

Dr. Ghartey is Assistant Professor, Maternal Fetal Medicine, Department of Women's Health, Dell Medical School/ University of Texas at Austin.

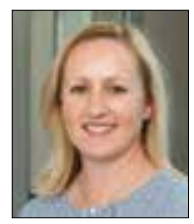

Dr. Cahill is Professor, Maternal Fetal Medicine, Department of Women's Health, Dell Medical School/ University of Texas at Austin.

The authors report no financial relationships relevant to this article.

doi: 10.12788 /obgm.0085 extensions and postpartum hemorrhage. The birth weight was $4,100 \mathrm{~g}$, and 5- and 10-minute Apgar scores were 4 and 8, respectively. The umbilical cord arterial $\mathrm{pH}$ was 7.03.

Ms. J. and her baby were discharged home on postoperative day 4 .

n 2014, the American College of Obstetricians and Gynecologists and the Society for Maternal-Fetal Medicine jointly released a document, "Safe prevention of the primary cesarean delivery," in response to the sharp rise in cesarean births from 1996 to $2011 .^{1}$ It described management strategies to safely reduce the most common indications for a primary CD in nulliparous women. Specifically, it recommended that the second stage of labor-defined as the interval from complete cervical dilation through delivery of the neonate-may be prolonged, as "longer durations may be appropriate on an individualized basis (eg, with the use of epidural analgesia or with fetal malposition) as long as progress is being documented." ${ }^{1}$

A prolonged second stage was defined as 3 hours of pushing in nulliparous women and 2 hours in multiparous women, with 1 additional hour (or longer) in those receiving epidural analgesia. Indeed, the primary CD rate decreased slightly to $21.7 \%$ in 2018 , down from $21.9 \%$ in $2017 .^{2}$ More recent evidence, however, has shown an increase in maternal and neonatal morbidity with prolonged second stage. ${ }^{3-8}$ 


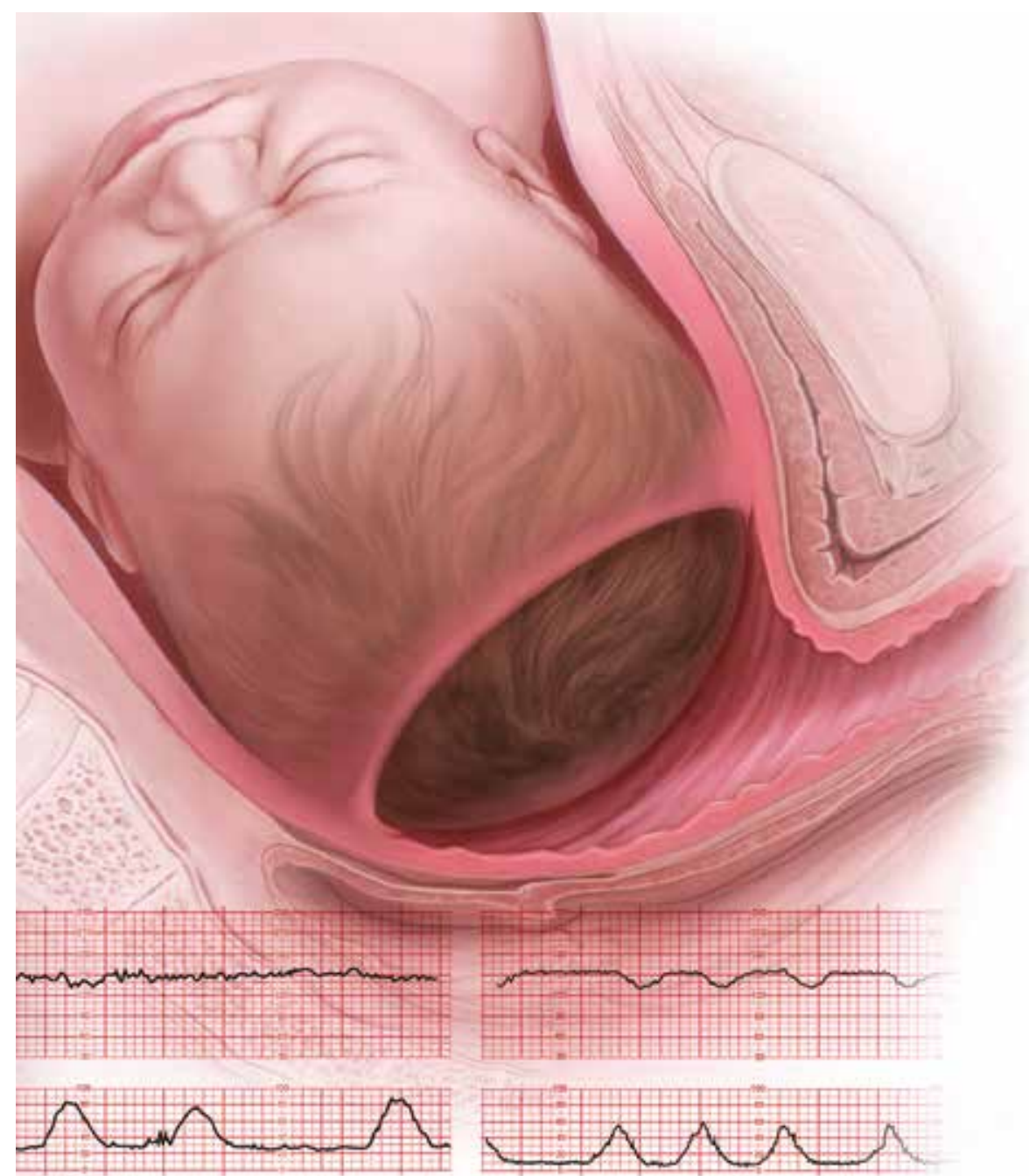

Efforts to manage the second stage from an evidence-based perspective are critical to balance the desired outcome of a safe vaginal delivery against the risks of prolonged second stage and operative vaginal delivery or CD.

\section{Perspectives on the "ideal" labor duration}

It is important to consider the historical context that led to the 2014 change in recommendations for duration of the second stage. ${ }^{9}$ In 1955, Dr. Emanuel Friedman published a prospective observational study of 622 consecutive primigravid parturients at term, of which 500 were included in the analysis that led to the graphicostatistical labor curve, or the well-known "Friedman's curve." ${ }^{10}$ The mean duration of the second stage was 0.95 hour. The statistical maximum for "ideal labor" for the second stage was set at 2 hours, with an additional hour allotted for patients receiving epidural analgesia.

In 2010, Zhang and colleagues published contemporary labor curves using data from the Consortium on Safe Labor, a multicenter retrospective observational study of 62,415 parturients. ${ }^{11}$ Among more than 25,000 nulliparous women, the median duration (95th percentile) of the second stage in hours was 1.1 (3.6), respectively. Notably, this analysis included only women with a spontaneous vaginal delivery and normal neonatal outcome.

Prior to the publication of the "Safe prevention of primary cesarean delivery," multiple investigations examined the relationship between the duration of the second stage and adverse maternal and neonatal outcomes, and the findings have been inconsistent. ${ }^{12-15}$

For example, Cheng and colleagues noted increased maternal complications that

\section{FAST \\ TRACK}

\section{Second stage management} requires a critical balance between the desired outcome of a safe vaginal delivery and the risk of prolonged second stage and operative vaginal delivery or $C D$ 
$\overline{\text { FAST }}$

TRACK

\section{Maternal factors \\ that influence}

the second stage

include parity, BMI, age, and clinical pelvimetry included postpartum hemorrhage, third- and fourth-degree perineal lacerations, and chorioamnionitis, but not neonatal morbidity, with each increasing hour within the second stage. ${ }^{12}$ By contrast, a large, population-based cohort study among low-risk women showed an increase in low 5-minute Apgar scores, admission to the neonatal intensive care unit (NICU), and composite perinatal morbidity with prolonged second stage. ${ }^{15}$ Furthermore, a secondary analysis of the Pushing Early or Pushing Late with Epidural (PEOPLE) trial showed that the chances of a vaginal delivery with a newborn without signs of asphyxia decreased significantly every hour after the first hour, and the risk of postpartum hemorrhage and intrapartum fever increased significantly after 2 hours of pushing. ${ }^{14}$

While these findings may represent the risks inherent with the intervention of operative delivery and not the duration of second stage of labor per se, one could posit that if the intervention were initiated earlier, could it prevent or at least reduce maternal and neonatal morbidity?

\section{Factors to assess and monitor in the second stage}

When assessing progress in the second stage of labor, consider:

- maternal factors

- fetal/neonatal factors, and

- modifiable factors.

Maternal factors that influence the second stage of labor include parity, body mass index (BMI), age, and clinical pelvimetry. ${ }^{11,16-19} \mathrm{Fetal} /$ neonatal factors that impact the second stage include the estimated fetal weight, fetal presentation (cephalic, face, and so on), position, and station, as well as the FHR Category. ${ }^{20,21}$ Factors that can be modified in the second stage include the effect of epidural analgesia (turning it down to reduce motor blockade while maintaining sensory pain relief so that patients feel the "urge" to push), maternal pushing position and technique, the presence of maternal support person(s), manual rotation for a fetal position that is not optimal, immediate versus delayed pushing, and prevention of perineal tears. ${ }^{22-32}$ Interestingly, epidural analgesia, parity, birth weight, and station at complete dilation predicted second stage duration but accounted for only $25 \%$ of the variability in second stage length, leaving $75 \%$ of the variance unexplained..$^{16}$

A specific absolute maximum length of time spent in the second stage of labor beyond which all women should undergo operative delivery has not been identified. ${ }^{1}$ Therefore, maternal, fetal/neonatal, and modifiable factors need to be critically assessed and continually monitored to determine whether a prolonged second stage or an operative delivery is warranted to prevent or minimize adverse maternal and neonatal outcomes.

\section{Maternal factors}

Maternal age correlates directly with the length of the second stage. That is, the length of the second stage increases with increasing age. ${ }^{17}$

Multiparous women have a shorter length of the second stage, regardless of epidural analgesia, compared with nulliparous women. ${ }^{11}$ In the Consortium for Safe Labor, multiparous women had a significantly shorter median second stage compared with nulliparous women. ${ }^{11}$

In adjusted analyses, maternal obesity was associated with an increased risk for $\mathrm{CD}$, with the risk of $\mathrm{CD}$ more than 3 times greater in women with a BMI higher than $40 \mathrm{~kg} / \mathrm{m}^{2}$ compared with those who had a BMI less than $25 \mathrm{~kg} / \mathrm{m}^{2} .^{18}$ There were no significant differences in the length of the second stage of labor by BMI catgeories. ${ }^{19}$

\section{Fetal factors}

Birth weight greater than 4,000 g was associated with an increased risk for arrest of descent during the second stage. ${ }^{33}$

Persistent fetal occiput posterior or transverse position may impact the duration of the second stage. A retrospective cohort study in women who underwent a trial of manual rotation compared with expectant management during the second stage of labor 
with the fetus in occiput posterior or occiput transverse position found that women with manual rotation were less likely to have a $\mathrm{CD}$, severe perineal laceration, postpartum hemorrhage, and chorioamnionitis. However, an increased risk of cervical laceration was associated with manual rotation. ${ }^{20}$

Regarding FHR status, FHR abnormalities occurred in $91 \%$ of second stage labor patterns, with Category II being the most common. ${ }^{21}$ The fetal status should remain reassuring to allow for continuation of the second stage.

\section{Epidural analgesia}

About $60 \%$ of women receive neuraxial analgesia in the United States, ${ }^{22}$ although rates vary widely across different populations. A Cochrane review showed no difference in the duration of the second stage among women who had early versus late initiation of epidural analgesia in labor. ${ }^{23}$ Epidural analgesia has no impact on the risk of $\mathrm{CD}$; however, women with epidural analgesia experienced more hypotension, motor blockade, fever, and urinary retention. ${ }^{24}$

One management practice has been to discontinue epidural analgesia to allow resumption of sensory and motor nerve function. Another Cochrane systematic review found no difference in mode of delivery or neonatal outcomes. ${ }^{25}$ Rather than discontinuing epidural analgesia, which results in a profound increase in inadequate pain relief, one may consider titrating the dose with joint patient decisionmaking to allow for greater motor capability while maintaining adequate analgesia. ${ }^{34}$

\section{Immediate vs delayed pushing}

The 2 most common approaches to managing the second stage were either to initiate pushing with contractions once complete dilation occurred (immediate pushing) or to allow for a rest period in which the fetus passively rotated and descended while conserving a woman's energy for pushing efforts (delayed pushing, laboring down, or passive descent). Since the publication of "Safe prevention of primary cesarean delivery," however, studies have shown a concerning association between maternal and neonatal complications and prolonged second stage (which may occur with delayed pushing). ${ }^{3-8,35}$ An observational study of nearly 44,000 nulliparous women without epidural analgesia found that prolonged second stage was associated with increased chorioamnionitis, third- and fourthdegree lacerations, neonatal sepsis, neonatal asphyxia, and perinatal mortality. ${ }^{35}$

A pragmatic multicenter randomized clinical trial on the optimal management of second stage of labor across the United States recently was conducted. ${ }^{7}$ More than 2,000 nulliparous women at term in spontaneous or induced labor with epidural analgesia were randomly assigned at complete dilation to immediate pushing or delayed pushing (1 hour after complete dilation). There was no difference in the rate of vaginal delivery. The rate of postpartum hemorrhage was significantly lower among women in the immediate-pushing group compared with the delayed-pushing group ( $2.3 \%$ vs $4.0 \%$, respectively; relative risk [RR], 0.6; 95\% confidence interval $[\mathrm{CI}], 0.3-0.9 ; P=.03$ ). Furthermore, rates of chorioamnionitis were significantly lower among women in the immediatepushing group compared with the delayedpushing group (6.7\% vs $9.1 \%$, respectively; RR, 0.70; 95\% CI, 0.66-0.90; $P=.005)$. No significant difference occurred in the composite outcome of neonatal morbidity between the groups. However neonatal acidemia (umbilical cord arterial $\mathrm{pH}<7.1$ ) and confirmed or suspected sepsis were significantly increased in the delayed-pushing group.

The evidence supports active pushing at the start of the second stage. Women who consider delayed pushing should be informed that delayed pushing has not been shown to increase the likelihood of vaginal birth and that it is associated with increased risks of infection, hemorrhage, and neonatal acidemia. ${ }^{36}$

\section{Maternal pushing position and technique}

Spontaneous pushing (in which women are free to follow their instincts and generally push 3 to 5 times per contraction) versus directed pushing (women are encouraged to

\section{FAST \\ TRACK}

The evidence supports active pushing at the start of the second stage 


\section{Key points for an evidence-based approach to the management of the second stage of labor}

- At the start of the second stage (complete dilation), patients should be encouraged to actively push.

- Reassessment should occur at regular intervals to assess for progress, after ensuring maternal and fetal well-being.

- An attempt at manual rotation or titration of epidural analgesia should be considered if there has been no advancement in fetal station.

- Additional considerations:

- Consider warm compresses or perineal massage to prevent third- and fourth-degree tears.

- The presence of a continuous support person may reduce the risk of an operative delivery.

- Delivery should be expected within 2 hours for multiparous women and 3 hours for nulliparous women in the second stage.

- Prolonging the second stage beyond these thresholds should be individualized and occur only in the setting of assured maternal and fetal well-being. improve outcomes for women and infants, including increased spontaneous vaginal birth, shorter duration of labor, and decreased cesarean birth. ${ }^{29}$ In a randomized trial of 412 healthy nulliparous women, women in labor were assigned to either a support group that received continuous support from a doula or an observed group that was monitored by an inconspicuous observer. Continuous labor support significantly reduced the rate of CDs and forceps deliveries. ${ }^{30,36}$ Indeed, during the COVID-19 pandemic, doulas have found innovative ways to continue to provide this essential support through virtual health. ${ }^{38}$

\section{Prevention of perineal tears}

Evidence suggests that warm compresses, and massage, may reduce third- and fourthdegree perineal tears. ${ }^{31} \mathrm{~A}$ meta-analysis of observational studies showed a significant reduction in the risk of OASI. ${ }^{32}$

take a deep breath at the beginning of a contraction then hold it and bear down throughout the contraction) demonstrated no clear difference in duration of the second stage perineal laceration, episiotomy, time spent pushing, or number of women with spontaneous vaginal birth. There was no difference in 5-minute Apgar score less than 7 or admission to the NICU. ${ }^{26}$

With regard to maternal positioning during the second stage, a Cochrane systematic review found benefits for upright posture, including a very small reduction in the duration of the second stage, reduction in episiotomy rates, and reduction in assisted deliveries. $^{37}$ There was an increased risk of blood loss greater than $500 \mathrm{~mL}$ and possibly an increased risk in second-degree tears. ${ }^{37} \mathrm{Com}$ pared with women allocated to lying down, women in the upright position during the second stage with epidural analgesia had significantly fewer spontaneous vaginal births. There was no difference in operative vaginal delivery, obstetric anal sphincter injury (OASI), infant Apgar score of less than 4 at 5 minutes, and maternal fecal incontinence at 1 year. ${ }^{28}$

\section{Maternal support person}

Continuous support during labor may

\section{Second stage steps: Recap}

Throughout the second stage of labor, the decision to continue with expectant management or intervene with either an operative vaginal delivery or a CD is complex and requires consistent assessment and integration of multiple factors. An evidence-based approach to second stage labor management includes active pushing that is either Valsalva pushing or spontaneous, coached or uncoached, but most importantly, at the start of the second stage when a patient reaches complete dilation. Reassessment should occur at regular intervals to determine progress, after ensuring maternal and fetal well-being.

If there has been no advancement in station, an attempt at manual rotation or titration of epidural analgesia should be considered. Importantly, fetal descent with adequate pushing should be demonstrated throughout the second stage.

Additional considerations that improve outcomes include warm compresses or perineal massage to prevent third- and fourthdegree tears and the presence of a continuous support person to reduce the risk for an operative delivery. 
Delivery should be expected within 2 hours for multiparous women and 3 hours for nulliparous women in the second stage. Prolonging the second stage beyond these thresholds should be individualized and occur only in the setting of assured maternal and fetal well-being.

\section{CASE An alternative management strategy}

Despite Ms. J.'s great active pushing effort for 60 minutes, the presenting part remains at 0 station and occiput transverse. Ms. J. is counseled regarding the risks and benefits of an attempt at manual rotation of the fetal head, and she wishes to proceed. The fetal position remains occiput transverse.
After another hour of active pushing, the FHR becomes Category II with repetitive variable decelerations. At this time, Ms. J. is informed that there has been no descent, and she is counseled on the risks and benefits of continued pushing versus $C D$. Through shared decision-making, she consents to a CD. She undergoes a primary $\mathrm{CD}$ without complication. The birth weight was $4,100 \mathrm{~g}$, and 5and 10-minute Apgar scores were 8 and 9, respectively. The umbilical cord arterial $\mathrm{pH}$ was 7.13.

Ms. J. and her baby were discharged home on postoperative day 4 .

\section{References}

1. American College of Obstetricians and Gynecologists Society for Maternal-Fetal Medicine; Caughey AB, Cahill AG, Guise JM, et al. Safe prevention of the primary cesarean delivery. Am J Obstet Gynecol. 2014;210:179-193. doi:10.1016/j. ajog.2014.01.026.

2. Martin JA, Hamilton BE, Osterman MJ, et al. Births: final data for 2018. Natl Vital Stat Rep. 2019;68:1-47.

3. Grobman WA, Bailit J, Lai Y, et al; Eunice Kennedy Schriver National Institute of Child Health and Human Development Maternal-Fetal Medicine Units Network. Association of the duration of active pushing with obstetric outcomes. Obstet Gynecol.2016;127:667-673.doi:10.1097/aog.0000000000001354.

4. Gimovsky AC, Berghella V. Randomized controlled tria of prolonged second stage: extending the time limit vs usual guidelines. Am J Obstet Gynecol. 2016;214:361.e1-6. doi:10.1016/j.ajog.2015.12.042.

5. Rosenbloom JI, Stout MJ, Tuuli MG, et al. New labor management guidelines and changes in cesarean delivery patterns. Am J Obstet Gynecol. 2017;217:689.e1-689.e8. doi:10.1016/j.ajog.2017.10.007.

6. Grantz KL, Sundaram R, Ma L, et al. Reassessing the duration of the second stage of labor in relation to maternal and neonatal morbidity. Obstet Gynecol. 2018;131:345-353. doi:10.1097/ aog.0000000000002431.

7. Cahill AG, Srinivas SK, Tita AT, et al. Effect of immediate vs delayed pushing on rates of spontaneous vaginal delivery among nulliparous women receiving neuraxial analgesia: a randomized clinical trial. JAMA. 2018;320:1444-1454. doi:10.1001/jama.2018.13986.

8. Zipori Y, Grunwald O, Ginsberg Y, et al. The impact of extending the second stage of labor to prevent primary cesarean delivery on maternal and neonatal outcomes. Am J Obstet Gynecol. 2019;220:191.e1-191.e7. doi:10.1016/j.ajog.2018.10.028.

9. Leveno KJ, Nelson DB, McIntire DD. Second-stage labor: how long is too long? Am J Obstet Gynecol. 2016;214:484-489. doi:10.1016/j.ajog.2015.10.926.

10. Friedman EA. Primigravid labor; a graphicostatistical analysis. Obstet Gynecol. 1955;6:567-589. doi:10.1097/00006250195512000-00001.

11. Zhang J, Landy HJ, Branch DW, et al. Contemporary patterns of spontaneous labor with normal neonatal outcomes. Obstet Gynecol. 2010;116:1281-1287. doi:10.1097/ AOG.0b013e3181fdef6e.

12. Cheng YW, Hopkins LM, Caughey AB. How long is too long: does a prolonged second stage of labor in nulliparous women affect maternal and neonatal outcomes? Am J Obstet Gynecol. 2004;191:933-938. doi:10.1016/j.ajog.2004.05.044.

13. Rouse DJ, Weiner SJ, Bloom SL, et al. Second-stage labor duration in nulliparous women: relationship to maternal and perinatal outcomes. Am J Obstet Gynecol. 2009;201:357.el-7. doi:10.1016/j.ajog.2009.08.003.
14. Le Ray C, Audibert F, Goffinet F, et al. When to stop pushing: effects of duration of second-stage expulsion efforts on maternal and neonatal outcomes in nulliparous women with epidural analgesia. Am J Obstet Gynecol. 2009;201:361.e1-7. doi:10.1016/j.ajog.2009.08.002.

15. Allen VM, Baskett TF, O'Connell CM, et al. Maternal and perinatal outcomes with increasing duration of the second stage of labor. Obstet Gynecol. 2009;113:1248-1258. doi:10.1097/ AOG.0b013e3181a722d6.

16. Piper JM, Bolling DR, Newton ER. The second stage of labor: factors influencing duration. Am J Obstet Gynecol. 1991;165(4 pt 1):976-979. doi:10.1016/0002-9378(91)90452-w.

17. Zaki MN, Hibbard JU, Kominiarek MA. Contemporary labor patterns and maternal age. Obstet Gynecol. 2013;122:1018-1024. doi:10.1097/AOG.0b013e3182a9c92c.

18. Kominiarek MA, Vanveldhuisen P, Hibbard J, et al; Consortium on Safe Labor. The maternal body mass index: a strong association with delivery route. Am J Obstet Gynecol. 2010;203:264.el-7. doi:10.1016/j.ajog.2010.06.024.

19. Kominiarek MA, Zhang J, Vanveldhuisen P, et al. Contemporary labor patterns: the impact of maternal body mass index. Am J Obstet Gynecol. 2011;205:244.el-8. doi:10.1016/j. ajog.2011.06.014.

20. Shaffer BL, Cheng YW, Vargas JE, et al. Manual rotation to reduce caesarean delivery in persistent occiput posterior or transverse position. J Matern Fetal Neonatal Med. 2011;24:6572. doi:10.3109/14767051003710276.

21. Krebs HB, Petres RE, Dunn LJ. Intrapartum fetal heart rate monitoring. V. Fetal heart rate patterns in the second stage of labor. Am JObstet Gynecol. 1981;140:435-439. doi:10.1016/00029378(81)90041-7.

22. Grant EN, Tao W, Craig M, et al. Neuraxial analgesia effects on labour progression: facts, fallacies, uncertainties and the future. BJOG. 2015;122:288-293. doi:10.1111/1471-0528.12966.

23. Sng BL, Leong WL, Zeng Y, et al. Early versus late initiation of epidural analgesia for labour. Cochrane Database Syst Rev. 2014;(10):CD007238. doi:10.1002/14651858.CD007238.pub2.

24. Anim-Somuah M, Smyth RM, Cyna AM, et al. Epidural versus non-epidural or no analgesia for pain management in labour. Cochrane Database Syst Rev. 2018;5(5):CD000331. doi:10.1002/14651858.CD000331.pub4.

25. Torvaldsen S, Roberts CL, Bell JC, et al. Discontinuation of epidural analgesia late in labour for reducing the adverse delivery outcomes associated with epidural analgesia. Cochrane Database Syst Rev. 2004;(4):CD004457. doi:10.1002/14651858.CD004457.pub2.

26. Lemos A, Amorim MM, Dornelas de Andrade A, et al. Pushing/bearing down methods for the second stage of labour. Cochrane Database Syst Rev. 2017;3(3):CD009124. doi:10.1002/14651858.CD009124.pub3.

27. Prins M, Boxem J, Lucas C, et al. Effect of spontaneous 
pushing versus Valsalva pushing in the second stage of labour on mother and fetus: a systematic review of randomised trials. BJOG. 2011;118:662-670. doi:10.1111/j.1471-0528.2011 $.02910 . \mathrm{x}$

28. Epidural and Position Trial Collaborative Group. Upright versus lying down position in second stage of labour in nulliparous women with low dose epidural: BUMPES randomised controlled trial. BMJ. 2017;359:j4471. doi:10.1136/bmj.j4471.

29. Bohren MA, Hofmeyr GJ, Sakala C, et al. Continuous support for women during childbirth. Cochrane Database Syst Rev. 2017;7(7):CD003766. doi:10.1002/14651858.CD003766.pub6.

30. Kennell J, Klaus M, McGrath S, et al. Continuous emotional support during labor in a US hospital. A randomized controlled trial. JAMA. 1991;265:2197-2201.

31. Aasheim V, Nilsen AB, Reinar LM, et al. Perineal techniques during the second stage of labour for reducing perineal trauma. Cochrane Database Syst Rev. 2017;6(6):CD006672. doi:10.1002/14651858.CD006672.pub3.

32. Bulchandani S, Watts E, Sucharitha A, et al. Manual perinea support at the time of childbirth: a systematic review and meta-analysis. BJOG. 2015;122:1157-1165. doi:10.1111/14710528.13431 .
33. Feinstein U, Sheiner E, Levy A, et al. Risk factors for arrest of descent during the second stage of labor. Int J Gynaecol Obstet. 2002;77:7-14. doi:10.1016/s0020-7292(02)00007-3.

34. Cheng YW, Caughey AB. Defining and managing normal and abnormal second stage of labor. Obstet Gynecol Clin North Am. 2017;44:547-566. doi:10.1016/j.ogc.2017.08.009.

35. Laughon SK, Berghella V, Reddy UM, et al. Neonatal and maternal outcomes with prolonged second stage of labor. Obstet Gynecol. 2014;124:57-67. doi:10.1097/ aog.0000000000000278.

36. American College of Obstetricians and Gynecologists. ACOG committee opinion no. 766: approaches to limit intervention during labor and birth. Obstet Gynecol. 2019;133:e164-e173. doi:10.1097/aog.0000000000003074.

37. Gupta JK, Sood A, Hofmeyr GJ, et al. Position in the second stage of labour for women without epidural anaesthesia. Cochrane Database Syst Rev. 2017;5(5):CD002006. doi:10.1002/14651858.CD002006.pub4.

38. Castaneda AN, Searcy JJ. Practising intimate labour: birth doulas respond during COVID-19. Anthropol Action. 2021;28:21-24. https://www.berghahnjournals.com/view/ journals/aia/28/1/aia280104.xml. Accessed February 8, 2021. 